\title{
Identification of Multivariable Models of Fast Ferries
}

\author{
J. Aranda ${ }^{1}$, J.M. de la Cruz ${ }^{2}$ and J.M. Díaz ${ }^{1}$ \\ ${ }^{1}$ Departmento de Informática y Automática, Facultad Ciencias, UNED, Madrid, Spain; ${ }^{2}$ Departmento de Arquitectura de Computadores y \\ Automática, Facultad Ciencias Físicas, Universidad Complutense, Madrid, Spain
}

This work presents the formal approach for identifying continuous transfer functions of the vertical dynamics of a high-speed ship as a nonlinear optimization problem with linear constraints. The proposed solution is described with a hybrid optimization method (genetic algorithm + nonlinear optimization algorithm with linear constraints from the Matlab toolbox).

Keywords: Identification Algorithm, Marine Systems; Optimization Problem

\section{Introduction}

Since the end of the Second World War the shipping industry has focused on using light metal alloys and plastic laminates to create units that are larger and more comfortable for passengers. A striking example of these kinds of ships is the so-called Fast Ferries, used on regular lines for transporting passengers and cars. The construction and exploitation of these kinds of vehicles is a growing market, with over 200 companies using 1250 fast ferries at present. In 2000 just in Europe, fast ferries transported 82.6 million passengers and 12.8 million cars.

Maritime transport's main competitor is air transport. Consequently, the keen interest shown by maritime passenger transport firms (shipping companies,

Correspondence to: J. Aranda, Departmento de Informática y Automática, Facultad Ciencias, UNED, Madrid, Spain. Fax: 34-91-398-66-97; Tel: 34-91-398-71-48. E-mail: jaranda@dia. uned.es ship owners and builders) in increasing their competitiveness with air transport is understandable.

One of the most unpleasant disadvantages of maritime transport is the motion sickness suffered by both passengers and crew as a result of the vertical accelerations associated with the induced heave and pitch motions. The decrease as far as possible in this motion sickness will lead to greater comfort and safety. In order to solve this problem using a control algorithm, first of all it is necessary to obtain a mathematical model of the process to be controlled.

There are two very different parts in the process model, on the one hand, the vertical dynamics of the high-speed ship and on the other hand, the mechanical actuators (fins, flaps, ...) that will be used to offset the heave and pitch motions.

In $[3,9,11,15,16]$, different transfer functions are proposed to describe the vertical dynamics of different kinds of ships. They are however not applicable to Fast Ferries. Moreover, in [8] the most appropriate models of mechanical actuators are described for reducing motion sickness in a Fast Ferry.

One possible way of obtaining the transfer functions of the vertical dynamics of a fast ferry is identification in the time or frequency domain; for this it is necessary to have the right experimental data. In the CEHIPAR (El Pardo Model Basin, Spain) sea behaviour experiments were done with a scaled down replica $(1 / 25)$ of a fast ferry from which experimental data in the form of time series of wave height, heave

Received 16 December 2002; Accepted 19 July 2003. Recommended by O. Egeland and M. Steinbuch. 
and pitch at different speeds (20, 30 and $40 \mathrm{kn}$ ) and different sea states $(\mathrm{SSN}=4,5$ and 6) were obtained.

The application of parametric identification techniques on the time series meant that it was possible to obtain ARX SISO and ARX SIMO models [5,7], that describe the ship's vertical dynamics according to the ship speed and sea state. These discrete models are splendid for gaining insight into the ship's vertical dynamics. Yet in order to use actuator elements to control the heave and pitch motions, it is necessary to decompose the dynamics into four models: heave force, pitch moment, heave motion and pitch motion.

The measurement of time series of heave force and the pitch moment [14] in a model basin is difficult and not very reliable. As a matter of fact, these magnitudes are not able to measure in a full scaled ship. Thus, the identification using discrete ARX type models of the heave force and the pitch moment was discarded.

However, it is possible to do an identification in the frequency domain since amplitude and phase data of the heave force, pitch moment and heave and pitch motions at different encounter frequencies and ship speed were obtained in CEHIPAR using the program PRECAL, which is based on a CAD description of the hull of the ship to be analysed. This program solves the physical equations of the vertical dynamics of a high-speed ship numerically using the Band Theory $[9,14,15]$. This theory basically consists of decomposing the ship's volume into narrow transversal bands on which the nonlinear differential equations of the ship's dynamics are solved by numerical integration.

This work presents the formal approach for identifying continuous transfer functions of the vertical dynamics of a high-speed ship as a nonlinear optimization problem with linear constraints. The proposed solution is described with a hybrid optimization method (genetic algorithm + nonlinear optimization algorithm with constraints from the Matlab toolbox).

\section{Initial Considerations}

To properly understand this work, it is necessary to describe and define the regular and irregular wave concepts, heave and pitch motions and encounter frequency.

\subsection{Wave Description}

Sea waves are principally caused by the interaction between the wind and water on the sea surface [14]. There are at least two physical processes involved here: the friction between the air and water, and the fields of local pressure associated with the blowing of the wind on the water. Although there are many works that try to explain the generation of waves by the wind $[13,19]$ nobody has developed a theory that thoroughly explains the energy transfer mechanism between the wind and sea.

Sea waves are usually irregular [15] both in time and space and more or less random by nature. If two waves are compared, it can be observed that they do not have exactly the same amplitude, they travel at different speeds and in different directions.

Regular waves never occur in a real maritime environment, although they can be easily generated in a laboratory with a model basin. Regular waves are used as the principal basis for many behaviour experiments of ships in a model basin, since they provide a lot of information about ship's vertical dynamics. Moreover, assuming linear wave theory is possible to consider that the right superposition of regular waves generates irregular waves.

Traditionally, sailors have used the visual appearance or sea state number (SSN) as an indication of the local speed of the wind. This has led to the numerical concept of sea state as a measurement of wave severity. Different scales of sea state have been developed by different national institutions. In 1970, the World Meteorological Organisation (WMO) established a code for describing sea states (see Table 1). Each SSN corresponds to a significant wave height range.

\subsection{Encounter Frequency}

Imagine a ship moving with a speed $U$, expressed in metres per second (the unit that sailors use to describe speed is the knot, which is equivalent to $0.51 \mathrm{~m} / \mathrm{s}$ ), on the sea surface (see Fig. 1).

Table 1. World Meteorological Organisation (WMO) sea state codes.

\begin{tabular}{lcl}
\hline SSN & $\begin{array}{l}\text { Significant wave height } \\
\text { range }(\mathrm{m})\end{array}$ & Description \\
\hline 0 & 0 & Calm (glassy) \\
1 & $0-0.1$ & Calm (rippled) \\
2 & $0.1-0.5$ & Smooth \\
3 & $0.50-1.25$ & Slight \\
4 & $1.25-2.5$ & Moderate \\
5 & $2.5-4.0$ & Rough \\
6 & $4.0-6.0$ & Very rough \\
7 & $6.0-9.0$ & High \\
8 & $9.0-14.0$ & Very high \\
9 & Over 14.0 & Phenomenal \\
\hline
\end{tabular}


A wave front moves with a constant speed $c$, the wavelength is $\lambda$. The waves fall against the ship forming an angle $\mu$ with the ship motion direction.

In Fig. 1, it can be seen that the component of the ship speed in the wave incidence direction is $U \cos \mu$, and that the waves will pass along the ship with a relative speed $c-U \cos \mu$.

Since the crests of the waves are separated by a distance $\lambda$, a crest will meet with the ship with an encounter period $T_{\mathrm{e}}$.

$$
T_{\mathrm{e}}=\frac{\lambda}{c-U \cos \mu} .
$$

The encounter frequency $\omega_{\mathrm{e}}$ is the relative frequency with which the waves fall against a moving ship, it is defined as:

$$
\omega_{\mathrm{e}}=\frac{2 \pi}{T_{\mathrm{e}}}=\frac{2 \pi}{\lambda}(c-U \cos \mu) .
$$

Or equivalently,

$$
\omega_{\mathrm{e}}=\omega-k U \cos \mu .
$$

In deep waters the number of waves have the form [15] $k=\omega^{2} / g$, where the expression $\omega_{\mathrm{e}}$ is:

$$
\omega_{\mathrm{e}}=\omega-\frac{\omega^{2}}{g} U \cos \mu .
$$

In a head sea $\mu=180^{\circ}$, the encounter frequency is expressed:

$$
\omega_{\mathrm{e}}=\omega+\frac{\omega^{2}}{g} U
$$

In other words, in a head sea the encounter frequency $\omega_{\mathrm{e}}$ is greater than the wave frequency $\omega$, and is directly proportional to the ship speed $U$.

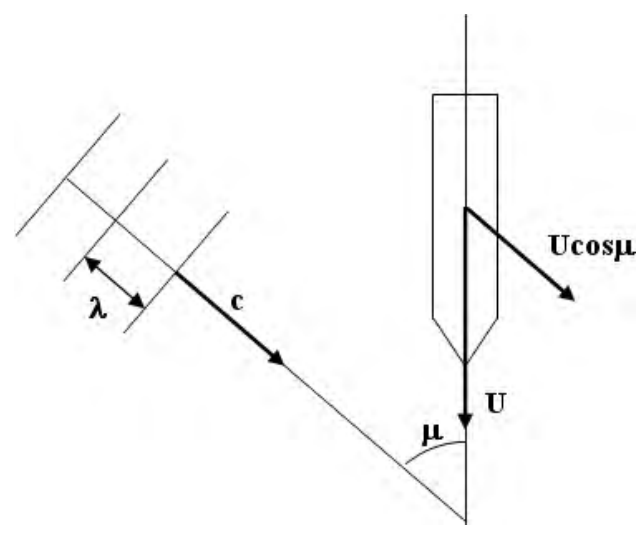

Fig. 1. Train of regular waves falling against a moving ship.

\section{Qualitative Description of the Vertical Dynamics of a High-Speed Ship}

A ship is a rigid solid partially submerged in water. As a result of the waves acting on the ship a series of forces and moments are induced that cause six different motions: surge, sway, heave, roll, pitch and yaw. There are physical equations that describe the six motions, they are nonlinear differential equations in terms of hydrodynamic forces and moments $[9,14,15]$.

Most ships have port/starboard symmetry and so the ship dynamic can be decomposed [15] into two planes:

- Horizontal plane, where the roll, yaw and sway motions are presented.

- Vertical plane, where the heave, pitch and surge motions are presented.

The interaction between the vertical and horizontal dynamics is usually small for high speed slender bodies, so that a good assumption $[14,15]$ is to consider both dynamics as independent. Furthermore, in head seas, i.e., waves that perpendicularly hit the ship bow, the vertical accelerations that cause motion sickness are exclusively associated with the heave and pitch motions.

Heave motion is understood to be the vertical displacement $h$ on the ship's centre of gravity as a result of the waves (see Fig. 2). Its unit of measurement is the metre.

Pitch motion is understood to be the angular displacement $p$ on the ship's vertical plane as a result of the waves (see Fig. 2). Its unit of measurement is the degree.

The sign convention that is used for the heave and pitch motion varies depending on the reference consulted. This work uses the following:

- The heave motion is considered positive $h>0$ if the vertical displacement of the centre of gravity is upwards.

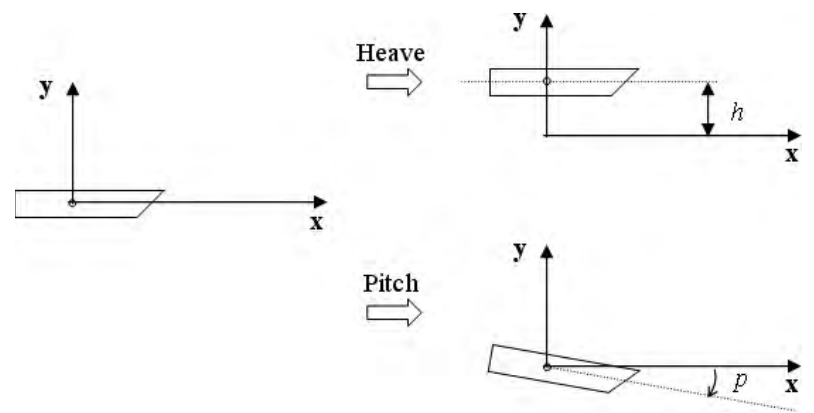

Fig. 2. Heave motion and pitch motion. 
- The pitch motion is considered positive $p>0$ if the bow sinks above the level of calm waters.

The heave and pitch motions are coupled, but this coupling is generally weak so in the first approximation they can be considered as independent motions [15].

Depending on the wavelength of the waves $\lambda$ and the ship's length $L$, three kinds of waves are distinguished (see Fig. 3): long (if $\lambda \gg L$ ), intermediate (if $\lambda \approx L)$ and short $(\lambda \ll L)$.

Suppose that large regular waves fall with a head sea on a ship. The time series of wave height $w$, heave $h$ and pitch $p$ can be expressed using the following equations:

$$
\begin{aligned}
w & =w_{0} \sin \left(\omega_{\mathrm{e}} t\right), \\
h & =h_{0} \sin \left(\omega_{\mathrm{e}} t+\delta_{\mathrm{h}}\right), \\
p & =p_{0} \sin \left(\omega_{\mathrm{e}} t+\delta_{\mathrm{p}}\right),
\end{aligned}
$$

where $w_{0}, h_{0}$ and $p_{0}$ are the amplitude of wave height, heave and pitch, respectively. $\omega_{\mathrm{e}}$ is the encounter frequency. $\delta_{\mathrm{h}}$ and $\delta_{\mathrm{p}}$ are the phase of heave and pitch, respectively.

Figure 4 represents for a fixed point the wave depression $w$ according to time. This graph also
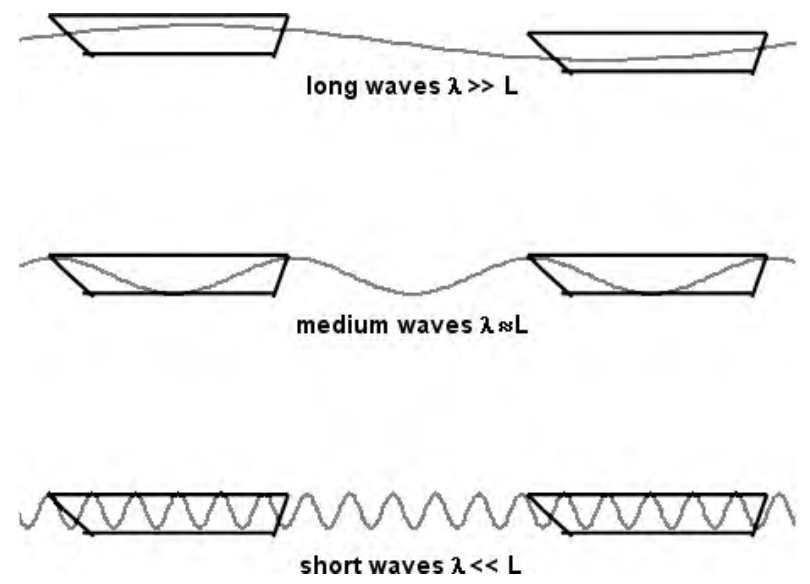

Fig. 3. Kinds of waves depending on the wavelength of the waves $\lambda$ and the ship's length $L$.

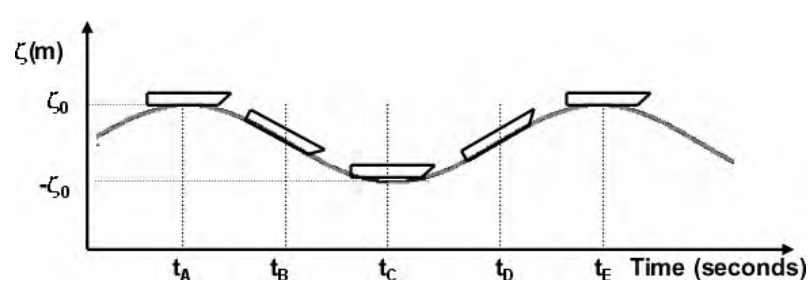

Fig. 4. Heave and pitch motion for large regular waves fall with a head sea on a ship. represents what the position of the ship would be. For the time instant $t_{\mathrm{A}}=\pi / 2 \omega_{\mathrm{e}} \mathrm{s}$, coinciding with a wave crest $\left(w=w_{0}\right)$ the maximum value of $\left(h=h_{0}\right)$ is presented. For the time instant $t_{\mathrm{B}}=\pi / \omega_{\mathrm{e}} \mathrm{s}$, coinciding with the cross through zero $(w=0)$ from a positive value to a negative value of wave depression the maximum value of pitch is obtained $\left(p=p_{0}\right)$. For the time instant $t_{\mathrm{C}}=(3 \pi / 2) / \omega_{\mathrm{e}} \mathrm{s}$, coinciding with a wave depression $\left(w=-w_{0}\right)$ the minimum heave value is obtained $\left(h=h_{0}\right)$. Finally, for the time instant $t_{\mathrm{D}}=(2 \pi) / \omega_{\mathrm{e}} \mathrm{s}$ coinciding with the cross through zero $(w=0)$ from a negative to a positive value of wave depression the minimum pitch value $\left(p=-p_{0}\right)$ is obtained.

Therefore, for these wave conditions it is concluded that the heave is synchronised $\left(\delta_{\mathrm{h}}=0^{\circ}\right)$ with the wave motion, while the pitch is dephased $\left(\delta_{\mathrm{p}}=-90^{\circ}\right)$ with the wave amplitude.

In order to know the asymptotic behaviour of the heave and pitch motions, suppose that there are two extremely long waves, which would correspond to the tide after several hours, i.e., the wave frequency tends towards zero $\omega \rightarrow 0$. In this instance the ship follows the surface of the water in such a way that the heave and wave amplitudes are equal, the pitch is also cancelled out. Therefore, the transfer functions wavesheave $(h(s) / w(s))$ and waves-pitch $(p(s) / w(s))$ tend towards 1 and 0 , respectively, when the wave frequency is decreasing

$$
\left|\frac{h(j \omega)}{w(j \omega)}\right| \underset{\omega \rightarrow 0}{\longrightarrow} 1, \quad\left|\frac{p(j \omega)}{w(j \omega)}\right| \underset{\omega \rightarrow 0}{\longrightarrow} 0 .
$$

Furthermore, for extremely short waves, i.e., the wave frequency tends towards infinite $\omega \rightarrow \infty$, there would be ripples to which the ship does not respond, so that there is neither heave nor pitch motion, which in terms of the transfer functions waves-heave $(h(s) / w(s))$ and waves-pitch $(p(s) / w(s))$ is expressed as follows:

$$
\left|\frac{h(j \omega)}{w(j \omega)}\right| \underset{\omega \rightarrow \infty}{\longrightarrow} 0, \quad\left|\frac{p(j \omega)}{w(j \omega)}\right| \underset{\omega \rightarrow \infty}{\longrightarrow} 0 .
$$

The asymptotic behaviors described by Eqs (7) and (8) must be borne in mind in the process for identifying transfer functions.

\section{Approach for Identification Problems}

Within the non-parametric identification techniques, one of the most commonly used is the frequency response. This method consists of measuring the system's response in the stationary state when a sinusoidal input is applied. The changes in amplitude and 
phase will determine the system's response to this frequency. If the experiment is repeated for a set of frequencies that include the system's bandwidth, there will be amplitude and phase measures according to frequency, which can be represented using a Bode diagram.

If the experimental points of the Bode diagram of a system are known, using an optimization problem solution it is possible to identify a continuous transfer function that presents a similar frequency response and that can therefore be considered as a good model of the system.

The vertical dynamics of a high-speed ship can be represented by the block diagram in Fig. 5, where

- $G_{\mathrm{H}}(s)$ is the transfer function where the input is the wave height $w(\mathrm{~m})$ and the output the heave motion $h(\mathrm{~m})$.

- $G_{\mathrm{P}}(s)$ is the transfer function where the input is the wave height $w(\mathrm{~m})$ and the output the pitch motion $p\left(^{\circ}\right)$.

Furthermore, the waves induce a heave force and a pitch moment in the ship, which cause the heave and pitch motions, respectively. Thus, the vertical dynamics can also be represented by the extended block in Fig. 6, where

- $G_{1 \mathrm{H}}(s)$ is the transfer function where the input is the wave height $w(\mathrm{~m})$ and the output the heave force $F_{\mathrm{H}}(\mathrm{KN})$.

- $G_{2 \mathrm{H}}(s)$ is the transfer function where the input is the heave force $F_{\mathrm{H}}(\mathrm{KN})$ and the output the heave motion $h(\mathrm{~m})$.

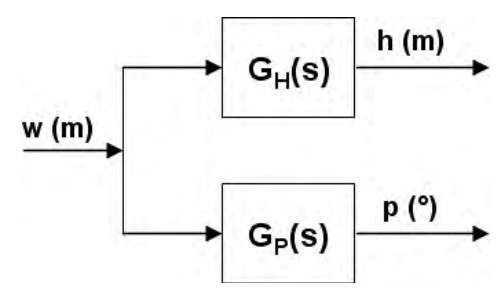

Fig. 5. Block diagram of the vertical dynamics of a highspeed ship.

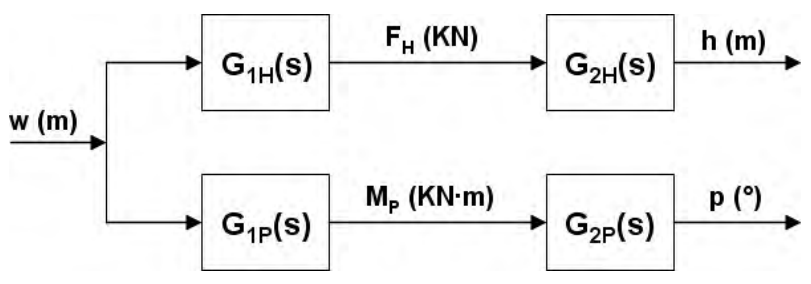

Fig. 6. Extended blocks diagram of the vertical dynamics of a high-speed ship.
- $G_{1 \mathrm{P}}(s)$ is the transfer function where the input is the wave height $w(\mathrm{~m})$ and the output the pitch moment $M_{\mathrm{P}}(\mathrm{KN} \mathrm{m})$.

- $G_{2 \mathrm{P}}(s)$ is the transfer functions where the input is the pitch moment $M_{\mathrm{P}}(\mathrm{KN} \mathrm{m})$ and the output the pitch motion $p\left(^{\circ}\right)$.

Therefore, the following relations are fulfilled:

$$
\begin{aligned}
G_{\mathrm{H}}(s) & =G_{1 \mathrm{H}}(s) \cdot G_{2 \mathrm{H}}(s), \\
G_{\mathrm{P}}(s) & =G_{1 \mathrm{P}} \cdot G_{2 \mathrm{P}}(s) .
\end{aligned}
$$

The identification of $G_{1 \mathrm{H}}(s)$ and $G_{1 \mathrm{P}}(s)$ is done directly, since there are data of amplitude and phase at different encounter frequencies for both transfer functions. These data were obtained using the software program PRECAL. However, the identification of $G_{2 \mathrm{H}}(s)$ and $G_{2 \mathrm{P}}(s)$ must be done indirectly, since there are no data for these transfer functions. Therefore the identification of $G_{1 \mathrm{H}}(s)$ and $G_{1 \mathrm{P}}(s)$ must be done first of all and the $G_{2 \mathrm{H}}(s)$ and $G_{2 \mathrm{P}}(s)$ expressions must be obtained from Eqs (9) and (10) by identifying on the $G_{\mathrm{H}}(s)$ and $G_{\mathrm{P}}(s)$ data.

Generally, suppose that there is a set of $N$ experimental points of amplitude $\left|G\left(j \omega_{\mathrm{e} i}\right)\right|$ and phase $\arg \left(G\left(j \omega_{\mathrm{e} i}\right)\right)$ for different encounter frequencies $\omega_{\mathrm{e} i}$, $i=1, \ldots, N$. The real and imaginary part of these points is given by:

$$
\begin{aligned}
& \operatorname{Re}\left(G\left(j \omega_{\mathrm{e} i}\right)\right)=\left|G\left(j \omega_{\mathrm{e} i}\right)\right| \cos \left(\arg \left(G\left(j \omega_{\mathrm{e} i}\right)\right)\right), \\
& \operatorname{Im}\left(G\left(j \omega_{\mathrm{e} i}\right)\right)=\left|G\left(j \omega_{\mathrm{e} i}\right)\right| \sin \left(\arg \left(G\left(j \omega_{\mathrm{e} i}\right)\right)\right) .
\end{aligned}
$$

Let the estimated transfer function $\hat{G}(s)$ be:

$$
\hat{G}(s)=\frac{b_{m+1} s^{m}+b_{m} s^{m-1}+\cdots+b_{1}}{s^{n}+a_{n} s^{n-1}+\cdots+a_{1}} .
$$

Its parameter vector $\theta$ is:

$$
\theta=\left[a_{1}, \ldots, a_{n}, b_{1}, \ldots, b_{m+1}\right]^{\mathrm{T}} .
$$

The structure $\hat{G}(s)$ of is denoted by $(m, n, n p s)$, where $m$ is the number of zeros, $n$ is the number of poles and $n p s$ is the number of simple poles.

We want to identify the structure and the parameters $\theta$ of the estimated transfer function $\hat{G}(s)$, in such a way that its amplitude $\left|\hat{G}\left(j \omega_{\mathrm{e} i}\right)\right|$ and its phase $\arg \left(\hat{G}\left(j \omega_{\mathrm{e} i}\right)\right)$ in the encounter frequencies $\omega_{\mathrm{e} i}, i=1, \ldots, N$ have the least possible error for the values $\left|G\left(j \omega_{\mathrm{e} i}\right)\right|$ and $\arg \left(G\left(j \omega_{\mathrm{e} i}\right)\right)$, respectively.

Therefore, in order to estimate $\hat{G}(s)$ an optimization problem has to be solved, where it is necessary to define an expression of the error or cost function. A possible choice, in accordance with $[1,18]$ of the cost 
function is:

$$
J(\theta)=J_{\text {real }}(\theta)+J_{\text {imag }}(\theta),
$$

where

$$
\begin{aligned}
J_{\text {real }}(\theta) & =\sum_{i=1}^{N}\left(\operatorname{Re}\left(G\left(j \omega_{\mathrm{e} i}\right)\right)-\operatorname{Re}\left(\hat{G}\left(j \omega_{\mathrm{e} i}\right)\right)\right)^{2} \\
J_{\text {imag }}(\theta) & =\sum_{i=1}^{N}\left(\operatorname{Im}\left(G\left(j \omega_{\mathrm{e} i}\right)\right)-\operatorname{Im}\left(\hat{G}\left(j \omega_{\mathrm{e} i}\right)\right)\right)^{2}
\end{aligned}
$$

In order to identify the continuous models of the vertical dynamics of a ship, it is necessary to identify $G_{1 \mathrm{H}}(s), G_{2 \mathrm{H}}(s), G_{1 \mathrm{P}}(s)$ and $G_{2 \mathrm{P}}(s)$.

Let $\left(m_{1}, n_{1}, n p s_{1}\right)$ be the $G_{1 \mathrm{H}}(s)$ or $G_{1 \mathrm{P}}(s)$ structure, the range of values permitted for this structure is bounded in set $E_{1}$.

$$
E_{1}=\left\{\begin{array}{l}
1 \leq m_{1} \leq m_{1 \max } \\
1 \leq n_{1} \leq n_{1 \max } \\
n p s_{1}<n_{1 \max }
\end{array}\right\}
$$

Let $\left(m_{2}, n_{2}, n p s_{2}\right)$ be the $G_{2 \mathrm{H}}(s)$ or $G_{2 \mathrm{P}}(s)$ structure, the range of values permitted for this structure is bounded in set $E_{2}$.

$$
E_{2}=\left\{\begin{array}{l}
1 \leq m_{2} \leq\left(m_{\max }-m_{1 \max }\right) \\
1 \leq n_{2} \leq\left(n_{\max }-n_{1 \max }\right) \\
n p s_{2}<\left(n_{\max }-n_{1 \max }\right)
\end{array}\right\}
$$

In accordance with the theoretical equations of a ship's vertical dynamics $[9,15], m_{1 \max }=3, m_{\max }=5$, $n_{1 \max }=4, n_{\max }=7$.

Moreover, in the identification process of the different transfer functions the a priori knowledge of the vertical dynamics must be considered, which results in the following constraints:

1. The model must be stable, ie, the transfer functions $G_{1 \mathrm{H}}, G_{2 \mathrm{H}}, G_{1 \mathrm{P}}, G_{2 \mathrm{P}}$ must not have positive poles.

2. At low frequencies the functions $G_{1 \mathrm{H}}(s)$ and $G_{2 \mathrm{H}}(s)$ verify $\left|G_{1 \mathrm{H}}\left(j \omega_{\mathrm{e} i}\right)\right| \underset{\omega_{\mathrm{e}} \rightarrow 0}{\longrightarrow} 1$ and $\left|G_{2 \mathrm{H}}\left(j \omega_{\mathrm{e} i}\right)\right| \underset{\omega_{\mathrm{e}} \rightarrow 0}{\longrightarrow} 1$, which leads to the following restriction on the coefficients $G_{1 \mathrm{H}}(s)$ and $G_{2 \mathrm{H}}(s)$ :

$$
\left|b_{1}\right|=\left|a_{1}\right| \text {. }
$$

3. At low frequencies the transfer frequency $G_{1 \mathrm{P}}(s)$ verifies $\left|G_{1 \mathrm{P}}\left(j \omega_{\mathrm{e} i}\right)\right| \underset{\omega_{\mathrm{e}} \rightarrow 0}{\longrightarrow} 0$, which leads to the following restriction on the coefficients $G_{1 \mathrm{P}}(s)$ :

$$
b_{1}=0 \text {. }
$$

Four nonlinear optimization problems with linear constraints therefore have to be solved for each of the speeds considered:

- Problem 1: Find the function $\hat{G}_{1 \mathrm{H}}(s) \in E_{1}$ which minimises the cost function $J(\theta)$, subject to the restriction (18).

- Problem 2: Find the function $\hat{G}_{2 \mathrm{H}}(s) \in E_{2}$ which minimises the cost function $J(\theta)$, subject to the restriction (18).

- Problem 3: Find the function $\hat{G}_{1 \mathrm{P}}(s) \in E_{1}$ which minimises the cost function $J(\theta)$, subject to the restriction (19).

- Problem 4: Find the function $\hat{G}_{2 \mathrm{P}}(s) \in E_{2}$ which minimises the cost function $J(\theta)$.

To solve Problems 2 and 4, it is necessary to first solve Problems 1 and 3.

\section{Solution to the Identification Problems}

Problems 1 and 2 present problems of convergence related to the initial values of the parameter vector that is usually necessary to start any optimization routine. Thus, a solution to avoid these convergence problems is to use a hybrid optimization method [2] formed by a genetic algorithm GA and the nonlinear optimization algorithm with linear constraints from the Matlab toolbox [4]. It has been checked in multiple cases $[6,10,12,17]$ that the use of a GA generally provides a solution quite close to the global optimum since they are characterised by their capacity to avoid local minimums. The fulfilment of this property however depends on the right choice of the GA parameters (population size, generations number, cross probability, mutation probability,...). Each optimization problem generally needs a different GA parameters configuration. Therefore, the solution obtained with the GA serves as a excellent initial value for the nonlinear optimization algorithm to avoid undesirable convergence problems.

Problems 3 and 4 are solved using the nonlinear optimization algorithm without GA (non-hybrid optimization method), since the random generation of the initial value of the parameter vector is sufficient to achieve the right algorithm convergence.

It is demonstrated both empirically and theoretically that GAs provide a reliable search for solutions to problems in complex spaces $[6,10,12,17]$. Although probabilistic rules intervene, GAs do not represent a blind search through space for solutions to a problem. The idea is that the information that is accumulated in the search space, completely unknown at the beginning, can direct the posterior search towards subspaces with expected improvement. It is important to highlight that the use of GAs does not ensure finding the 
global optimum of a problem, but they may provide quite close solutions to it, since they are characterised by their capacity to avoid local minimums.

Since GAs solve maximisation problems, the cost function $J$ defined in Eq. (14) is transformed into:

$$
J_{\mathrm{G}}=\frac{100}{J} .
$$

The maximum of $J_{\mathrm{G}}$ coincides with the minimum of $J$. A factor of 100 included to increase the comparison range of the elements that constitute each generation in a GA population. The GA will inspect the spaces of structures $E_{1}$ and $E_{2}$ of $G_{1 \mathrm{H}}$ and $G_{2 \mathrm{H}}$, respectively, calculating the parameter vector for each one and selecting the one that presents the best cost function $J_{\mathrm{G}}$.

The functions selected with GA are denoted by $G_{1 \mathrm{HG}}(s)$ and $G_{2 \mathrm{HG}}(s)$, and they will be used as initial values to identify $G_{1 \mathrm{H}}(s)$ and $G_{2 \mathrm{H}}(s)$ by a nonlinear optimization algorithm with constraints from the Matlab toolbox.

The GA specifications [1] used are:

- Initial population of 10 individuals created at random.

- Substitution by generation.

- Probability of cross $p_{\mathrm{c}}=0.7$.

- Probability of mutation $p_{\mathrm{m}}=0.007$.

- The choice of parents is done using the roulette method.

- 10,000 generations run in 40 eras.

In order to ensure the stability of the estimated models using the nonlinear optimization method, the transfer function to be estimated has been expressed thus:

$\hat{G}(s)=\frac{x_{n+m+1} s^{m}+x_{n+m} s^{m-1}+\cdots+x_{n+1}}{\left(\prod_{i=1}^{n p c} A_{i}(s)\right)\left(s+x_{n p c+1}\right) \cdots\left(s+x_{n p c+n p s}\right)}$,

where

$$
A_{i}=\left[s^{2}+2 s x_{i-1}+\left(x_{i-1}^{2}+x_{i}^{2}\right)\right] \quad i=1, \ldots, n p c .
$$

The denominator has been rewritten in quadratic factors that represent the conjugate complex pole and in simple factors that represent the poles on the real axis. Therefore, given a total number of $n$ poles, there will be $n p c$ complex poles and $n p s$ simple poles.

The $\hat{G}(s)$ numerator does not factorise since it is the optimization algorithm which, given the number of total zeros $m$ of the structure, determines the right number of complex and simple zeros.
With a structure $(m, n, n p s)$ for the transfer function $\hat{G}(s)$, the parameter vector to be estimated is:

$$
\theta=\left[x_{1}, x_{2}, \ldots, x_{n p c-1}, x_{n p c}, x_{n p c+1}, \ldots, x_{n p c+n p s}\right]^{\mathrm{T}}
$$

If $\hat{G}(s)$ is written in the form (21), then the stability necessarily ensures that the real parts of all the poles are less than zero, i.e., they are in the left semiplane of plane $s$.

$$
x_{i}<0, \quad i, \ldots, n p c+n p s .
$$

For numerical calculations the restriction that is in fact used is:

$$
x_{i}<-0.005, \quad i=1, \ldots, n p c+n p s .
$$

The algorithm ident_G1 was developed to identify $\hat{G}_{1 \mathrm{H}}(s)$ or $\hat{G}_{1 \mathrm{P}}(s)$ using the non-linear optimization method, this algorithm was implemented as a function $\mathrm{M}$ of Matlab [7].

This algorithm works with two different representations of the transfer function to be estimated $\hat{G}(s)$ : Eq. (21) and the following equation:

$$
\hat{G}(s)=\frac{y_{m+n+1} s^{m}+y_{m+n} s^{m-1}+\cdots+y_{n+1}}{s^{n}+y_{n} s^{n-1}+\cdots+y_{1}} .
$$

The ident_G1 algorithm consists of the following steps:

1. Obtaining the initial values. The algorithm has to be given the structure $(m, n, n p s)$ of the transfer function to be estimated $\hat{G}(s)$, as well as an initial parameter vector $\theta_{0}$ of $\hat{G}(s)$. These initial values can be obtained with GA or be generated randomly. The algorithm also has to be given the $N$ data of the module $\left|G\left(j \omega_{\mathrm{e} i}\right)\right|$ and the phase $\arg \left(G\left(j \omega_{\mathrm{e} i}\right)\right) i=1, \ldots, N$, at different encounter frequencies of the function to be identified $G(s)$.

2. Normalisation of the data $\left|G\left(j \omega_{\mathrm{e} i}\right)\right|$ in order to avoid numerical calculation problems.

3. Calculation of the real part $\operatorname{Re}\left(G\left(j \omega_{\mathrm{e} i}\right)\right)$ and the imaginary part $\operatorname{Im}\left(G\left(j \omega_{\mathrm{e} i}\right)\right)$ from the data $\left|G\left(j \omega_{\mathrm{e} i}\right)\right|$ and $\arg \left(G\left(j \omega_{\mathrm{e} i}\right)\right)$.

4. Generation of the equations in symbolic form of the real part $\operatorname{Re}\left(\hat{G}\left(j \omega_{\mathrm{e} i}\right)\right)$ and the imaginary part $\operatorname{Im}\left(\hat{G}\left(j \omega_{\mathrm{e} i}\right)\right)$ of the transfer function to be estimated. Thus, Eq. (26) is written as follows:

$$
\begin{aligned}
& \hat{G}\left(j \omega_{\mathrm{e}}\right) \\
& \quad=\frac{\left(y_{n+1}-\omega_{\mathrm{e}}^{2} \cdot y_{n+3}+\cdots\right)+j\left(\omega_{\mathrm{e}} \cdot y_{n+2}-\omega_{\mathrm{e}}^{3} \cdot y_{n+4}+\cdots\right)}{\left(y_{1}-\omega_{\mathrm{e}}^{2} \cdot y_{3}+\cdots\right)+j\left(\omega_{\mathrm{e}} \cdot y_{2}-\omega_{\mathrm{e}}^{3} \cdot y_{4}+\cdots\right)}
\end{aligned}
$$


Or equivalently,

$$
\begin{aligned}
\hat{G}\left(j \omega_{\mathrm{e}}\right)= & \frac{n 1+j \cdot n 2}{d 1+j \cdot d 2}, \\
\hat{G}\left(j \omega_{\mathrm{e}}\right)= & H_{1}\left(y_{1}, \ldots, y_{m+n+1}, \omega_{\mathrm{e}}\right) \\
& +j H_{2}\left(y_{1}, \ldots, y_{m+n+1}, \omega_{\mathrm{e}}\right) .
\end{aligned}
$$

Consequently,

$$
\begin{aligned}
& \operatorname{Re}\left(\hat{G}\left(j \omega_{\mathrm{e}}\right)\right)=H_{1}\left(y_{1}, \ldots, y_{m+n+1}, \omega_{\mathrm{e}}\right), \\
& \operatorname{Im}\left(\hat{G}\left(j \omega_{\mathrm{e}}\right)\right)=H_{2}\left(y_{1}, \ldots, y_{m+n+1}, \omega_{\mathrm{e}}\right) .
\end{aligned}
$$

5. Generation of the coefficients in symbolic form of the numerator $\hat{G}$, applying accordingly, the constraints at low frequency defined in Eqs. (18) or (19).

6. Calculation of the symbolic expressions of the coefficients $y_{k}, k=1, \ldots, n$ of the denominator of $\hat{G}$ in its form (26), according to the coefficients $x_{k}$ of the denominator of $\hat{G}$ in its factorized form (21). In other words, the following kinds of equations are obtained:

$$
y_{k}=F_{k}\left(x_{1}, \ldots, X_{n}\right), \quad k=1, \ldots, n .
$$

7. Construction of the cost function $J(\theta)$ that is defined in (14).

8. Search for the optimum parameter vector $\theta_{\mathrm{opt}}$ that minimises the cost function $J$ using the non-linear optimization method with linear constraints from the Matlab toolbox (function constr.m).

Therefore the algorithm ident_G1, in a first stage (steps 1-7) appropriately constructs the cost function $J(\theta)$ to be optimised, considering the magnitude and phase experimental points of a function $G(s)$, the structure of the transfer function to be estimated $\hat{G}(s)$ and the constraints imposed on the values of its coefficients. Subsequently in a second stage (step 8) an optimization routine of the function $J(\theta)$ is executed using the non-linear optimization method. Thus the parameter vector $\theta_{\text {opt }}$ is obtained that minimises the cost function.

Furthermore, the algorithm ident_G2 was development to obtain the expressions $\hat{\bar{G}}_{2 \mathrm{H}}(s)$ or $\hat{G}_{2 \mathrm{P}}(s)$ from Eqs (9) and (10), identifying on the data of $G_{\mathrm{H}}(s)$ or $G_{\mathrm{P}}(s)$, respectively. In order to be able to apply the algorithm, it is necessary to have first obtained $\hat{G}_{1 \mathrm{H}}(s)$ or $\hat{G}_{1 \mathrm{P}}(s)$ with the algorithm ident_G1.

The steps for the algorithm ident_G2 are very similar to the steps for the algorithm ident_G1; it simply works with a two-transfer function product instead of with just one transfer function.

\subsection{Identification of $G_{1 \mathrm{H}}$ and $G_{2 \mathrm{H}}$}

The solution to Problems 1 and 2 for the identification of $G_{1 \mathrm{H}}$ and $G_{2 \mathrm{H}}$, was done following the optimization scheme illustrated in Fig. 7. This scheme consists of four steps:

1. Identification of an initial model $G_{1 \mathrm{HG}}$ using GA.

2. Identification of an initial model $G_{2 \mathrm{HG}}$ with GA, using the model $G_{1 \mathrm{HG}}$.

3. Identification of $G_{1 \mathrm{H}}$ with the algorithm ident_G1, taking as the initial value for the calculation the model $G_{1 \mathrm{HG}}$ obtained in step 1 .

4. Identification of the model $G_{2 \mathrm{H}}$ with the algorithm ident_G2, taking as the initial value $G_{2 \mathrm{HG}}$ and using the model $G_{1 \mathrm{H}}$ obtained in the previous step.

\subsection{Identification of $G_{1 \mathrm{P}}$ and $G_{2 \mathrm{P}}$}

The solution to Problems 3 and 4 was done in accordance with the optimization scheme illustrated in Fig. 8. This scheme consists of four steps:

1. Configuration of an initial model $G_{1 \mathrm{P} 0}$, for this a structure $\left(m_{1}, n_{1}, n p s_{1}\right) \in E_{1}$ is fixed and a parameter vector $\theta$ is generated randomly for the transfer function.

2. Identification of $G_{1 \mathrm{P}}$ with the algorithm ident_G1, using as the initial value the model $G_{1 \mathrm{P} 0}$.

3. Configuration of an initial model $G_{2 \mathrm{P} 0}$, for this a structure $\left(m_{2}, n_{2}, n p s_{2}\right) \in E_{2}$ is fixed and a parameter vector $\theta$ is generated randomly for the transfer function.

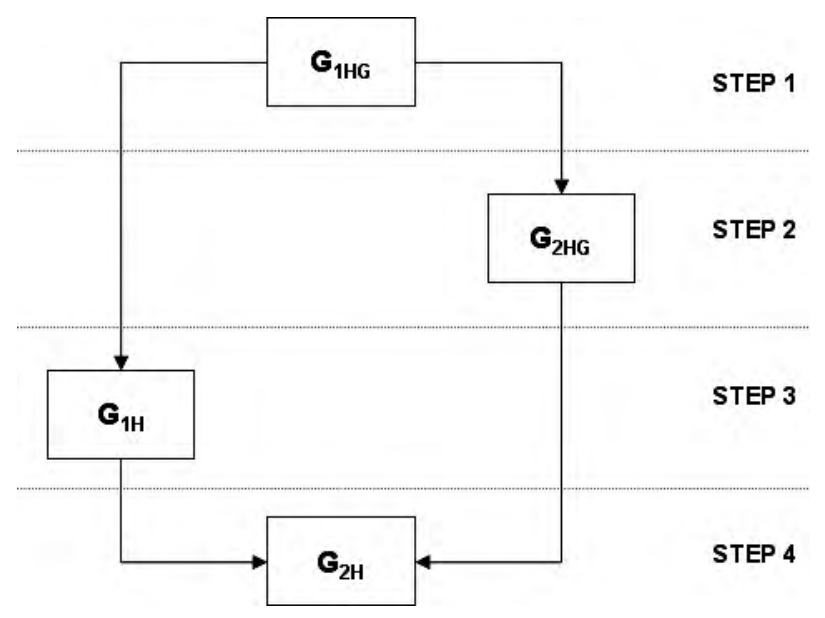

Fig. 7. Optimization scheme to identify $G_{1 \mathrm{H}}(s)$ and $G_{2 \mathrm{H}}(s)$. 
4. Identification of the model $G_{2 \mathrm{P}}$ with the ident_G2, taking as the initial value $G_{2 \mathrm{P} 0}$ and using the model $G_{1 \mathrm{P}}$ obtained in step 2 .

\section{Results Obtained and Validation}

The amplitude and phase data of the transfer function $G_{1 \mathrm{P}}, G_{1 \mathrm{H}}, G_{\mathrm{P}}$ and $G_{\mathrm{H}}$ were used for the identification of continuous models of the pitch moment, heave force, heave and pitch motions. These data were obtained in CEHIPAR with the help of the software program PRECAL at different encounter frequencies.

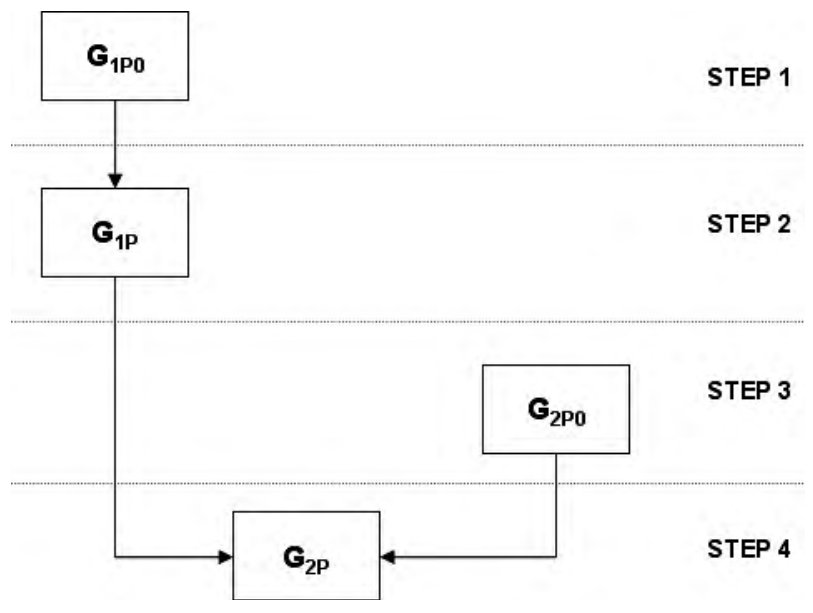

Fig. 8. Optimization scheme to identify $G_{1 \mathrm{P}}(s)$ and $G_{2 \mathrm{P}}(s)$.
The transfer functions $G_{1 \mathrm{H}}, G_{2 \mathrm{H}}, G_{1 \mathrm{P}}$ and $G_{2 \mathrm{P}}$ were identified for the speeds $U=20,30$ and $40 \mathrm{kn}$ [7]. For $U=40 \mathrm{kn}$, a case of special interest for navigation was obtained:

$$
\begin{aligned}
& G_{1 \mathrm{H}}(s)=\frac{0.067 s^{3}+0.102 s^{2}+0.904 s-0.189}{s^{4}+1.340 s^{3}+2.266 s^{2}+0.867 s+0.189}, \\
& G_{2 \mathrm{H}}(s)=\frac{0.267 s^{2}-0.510 s+3.053}{s^{2}+0.469 s+3.053}, \\
& G_{1 \mathrm{P}}(s)=\frac{-0.076 s^{3}+0.030 s^{2}-0.925 s}{s^{4}+3.029 s^{3}+6.194 s^{2}+6.854 s+2.706}, \\
& G_{2 \mathrm{P}}(s)=\frac{-2.104 s^{2}+16.440 s+0.691}{s^{3}+1.203 s^{2}+3.155 s+1.334} .
\end{aligned}
$$

The validation of the estimated transfer functions was done by representing their frequency response in a Bode diagram where they were compared with the amplitude and phase points obtained with PRECAL. In the case of $G_{2 \mathrm{H}}$ and $G_{2 \mathrm{P}}$ since there are no data, they are multiplied by $G_{1 \mathrm{H}}$ and $G_{1 \mathrm{P}}$, respectively, in such a way that the Bode that is represented is the wavesheave $G_{\mathrm{H}}$ and waves-pitch $G_{\mathrm{P}}$ filter. Figures $9-12$ show the Bode diagram of $G_{1 \mathrm{H}}(s), G_{\mathrm{H}}(s), G_{1 \mathrm{P}}(s)$ and $G_{\mathrm{P}}(s)$ compared with the amplitude and phase points obtained with PRECAL.

Moreover, the total continuous estimated model $G_{\mathrm{H}}(s)$ or $G_{\mathrm{P}}(s)$ is excited with irregular waves
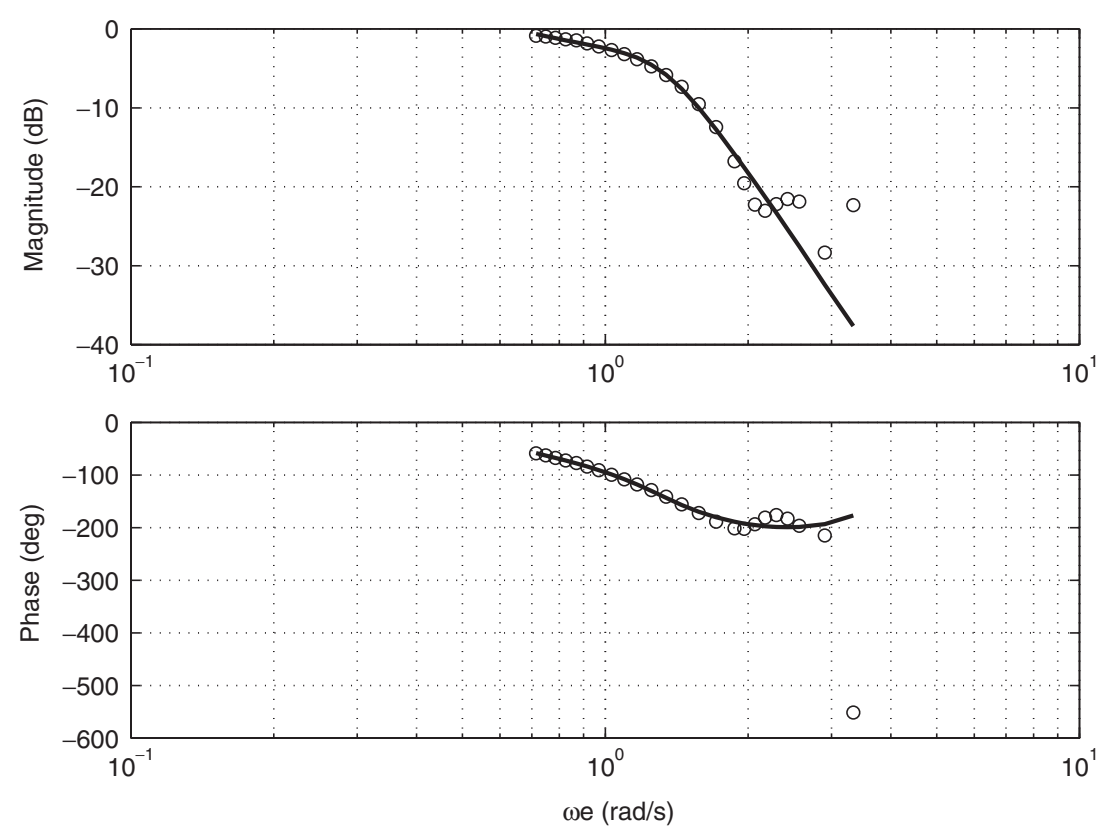

Fig. 9. Bode diagram of $G_{1 \mathrm{H}}(s)$ compared with the PRECAL amplitude and phase points. 

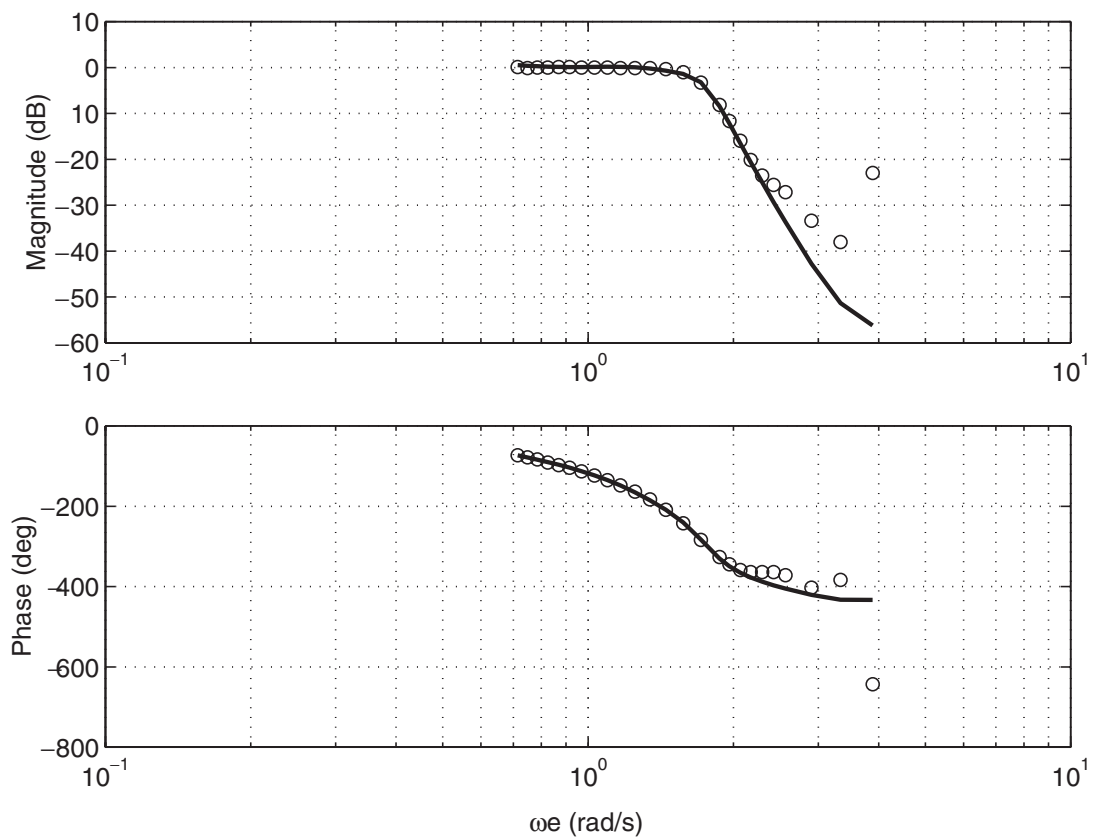

Fig. 10. Bode diagram of $G_{\mathrm{H}}(s)$ compared with the PRECAL amplitude and phase points.
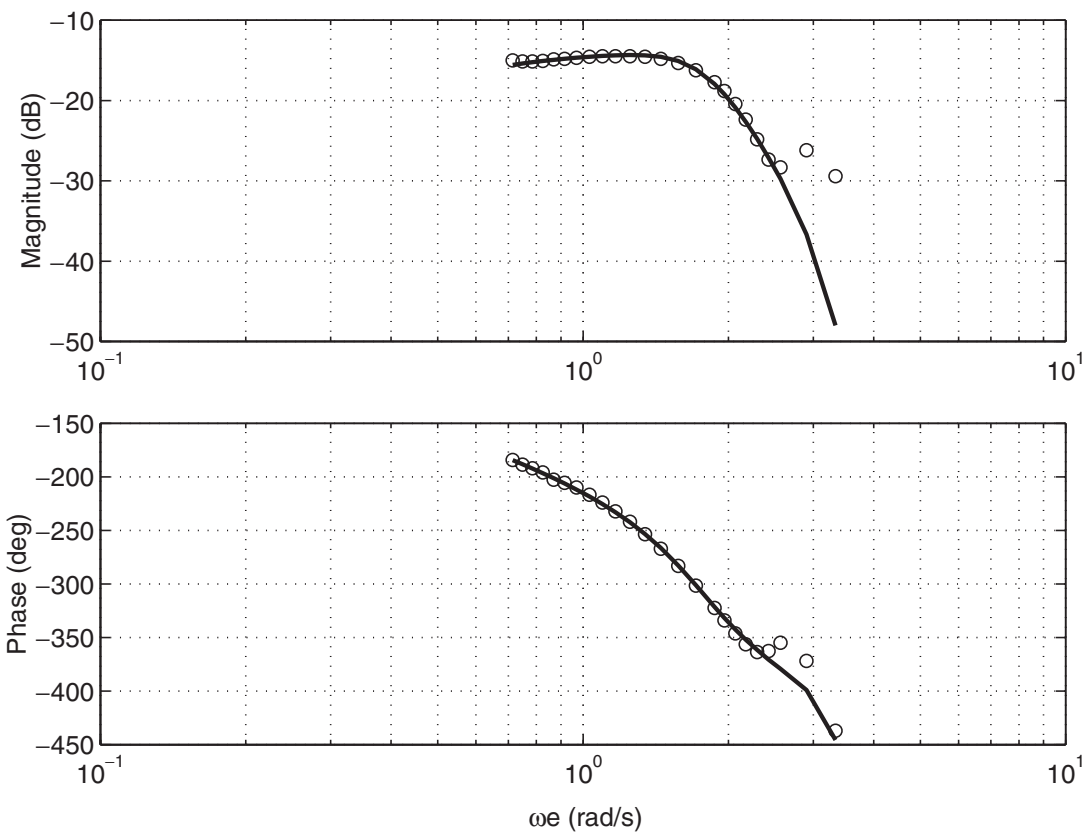

Fig. 11. Bode diagram of $G_{1 \mathrm{P}}(s)$ compared with the PRECAL amplitude and phase points.

measured experimentally in the CEHIPAR for a specific speed. The output generated by the model is represented with the measurement experimentally, also calculating the average quadratic error. In Fig. 13, the output $G_{\mathrm{H}}(s)$ is shown compared with the heave measured experimentally when the excitation input is irregular waves $\mathrm{SSN}=5$ to $U=40 \mathrm{kn}$. An average quadratic error $e 2 m=0.011$ is obtained.

In Fig. 14, the output $G_{\mathrm{P}}(s)$ is shown compared with the pitch measured experimentally when the excitation input is irregular waves $\mathrm{SSN}=5$ to $U=40 \mathrm{kn}$. An average quadratic error $e 2 m=0.1225$ is obtained. 

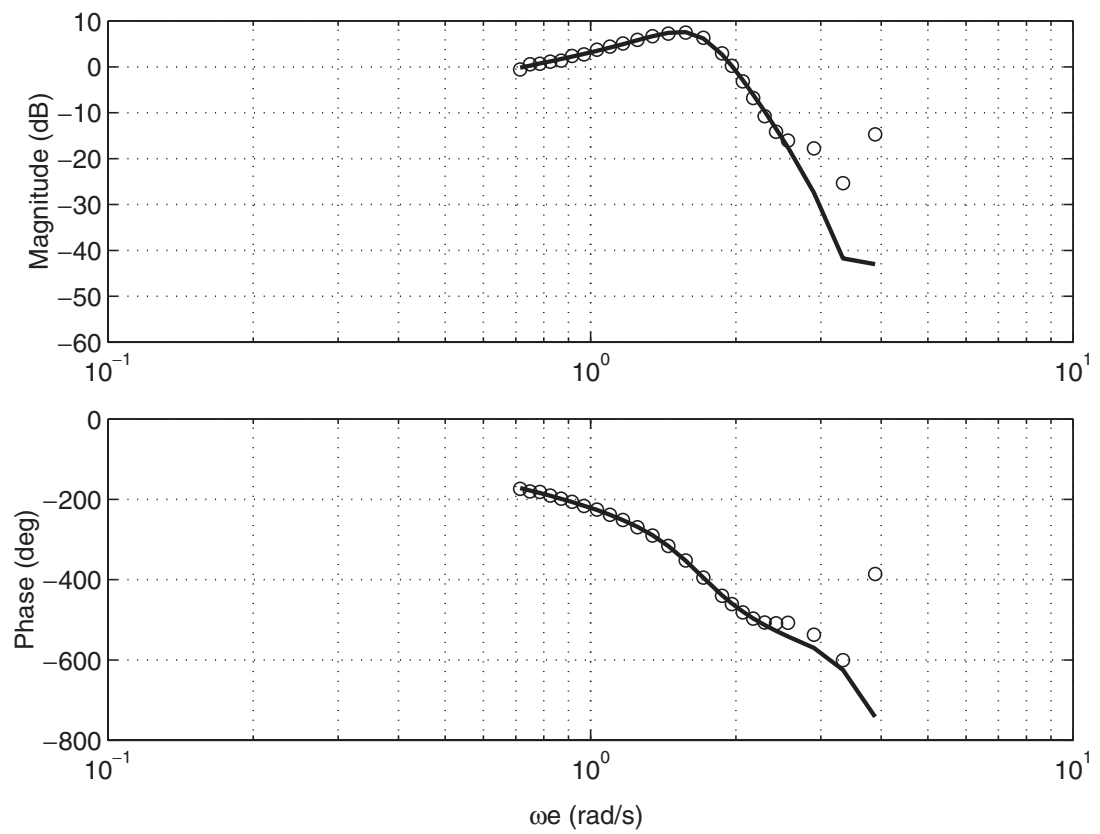

Fig. 12. Bode diagram of $G_{\mathrm{P}}(s)$ compared with the PRECAL amplitude and phase points.

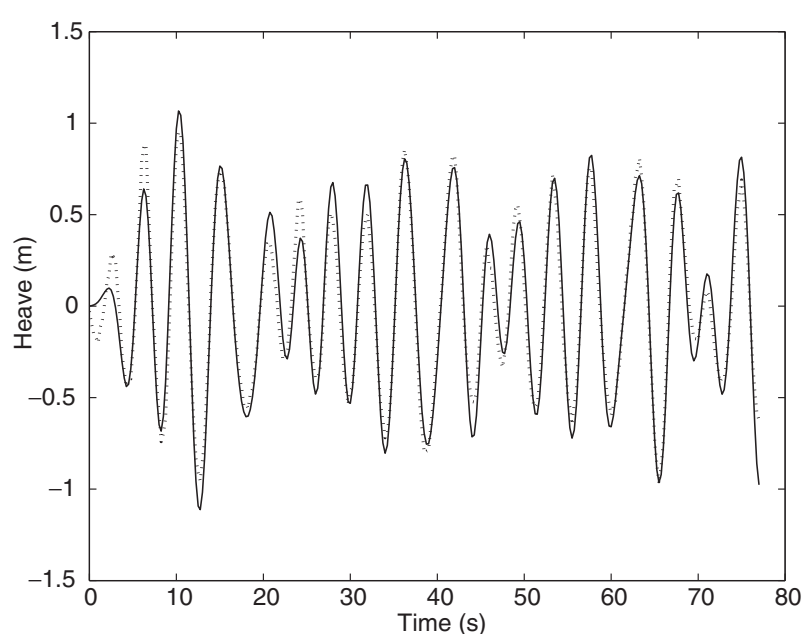

Fig. 13. The output of $G_{\mathrm{H}}(s)$ (solid line) is shown compared with the heave measured (broken line) experimentally when the excitation input is irregular waves $\mathrm{SSN}=5$ to $U=40 \mathrm{kn}$.

\section{Conclusions}

This work has presented identification in the domain of the frequency of continuous models of the vertical dynamics of a high-speed ship. The system has been decomposed into four filters: $G_{1 \mathrm{H}}, G_{1 \mathrm{P}}, G_{2 \mathrm{H}}$ and $G_{2 \mathrm{P}}$, to model the heave force, the pitch moment, heave and pitch respectively. In the identification process of the filters $G_{1 \mathrm{H}}$ and $G_{2 \mathrm{H}}$, a hybrid optimization method has been used formed by a genetic algorithm GA and a

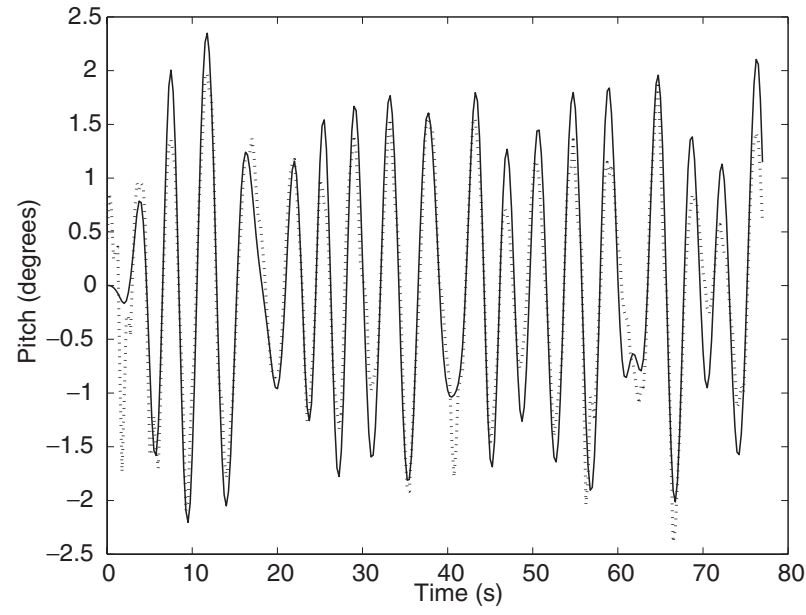

Fig. 14. The output of $G_{\mathrm{P}}(s)$ (solid line) is shown compared with the pitch measured (broken line) experimentally when the excitation input is irregular waves $\mathrm{SSN}=5$ to $U=40 \mathrm{kn}$.

nonlinear optimization algorithm with constraints from the Matlab toolbox. While in the identification of the filters $G_{1 \mathrm{P}}$ and $G_{2 \mathrm{P}}$, the nonlinear optimization algorithm without GA (non-hybrid optimization method) has been only used.

For the application of the nonlinear optimization method two algorithms in Matlab were developed: ident_G1 for the identification of $G_{1 \mathrm{H}}$ and $G_{1 \mathrm{P}}$, and ident_G2 for the identification of $G_{2 \mathrm{H}}$ and $G_{2 \mathrm{P}}$.

The main difference between the non-hybrid method and the hybrid method of identification is the 
choice of the initial values of the parameters to be identified from the transfer function structure used as the model. In the hybrid method where a GA is used first, some excellent initial values are achieved after a considerable calculation time. These initial values are close to the global optimum, so it takes very few iterations for the nonlinear optimization algorithm to reach the global optimum. In the non-hybrid method, the initial values are chosen randomly without wasting calculation time but the nonlinear optimization algorithm can require a greater number of iterations to converge to the global optimum. In fact, it may not even converge or just converge to a local optimum.

The experience acquired in the identification of the continuous models indicates that in models with not many parameters it is recommendable to use the nonhybrid method, because although it uses some random initial values it does not take long to converge to the global optimum, so it is not worth having a calculation time first to obtain good initial values using GA. While in models with many parameters the hybrid method is highly recommendable to avoid problems of convergence.

Bearing in mind the characteristics of both methods, in the solution to Problems 3 and 4 the nonhybrid method was used because the random generation of the initial value of the parameter vector $\theta$ was sufficient to achieve the right convergence of the nonlinear optimization algorithm. However for the solution to Problems 1 and 2 the hybrid method was preferred to avoid problems of convergence.

The continuous models that have been identified can be considered as good since they fulfil the requirements indicated in the validation process to which they were subjected, i.e., they present a good adjustment in the frequency domain to the amplitude and phase data obtained with PRECAL, and moreover their time simulations both with regular and irregular waves when compared with the experimental time series present a small average quadratic error.

\section{Acknowledgements}

This development was supported by CICYT of Spain under contracts DPI2000-0386-C03-01 and TAP970607-C03-02

\section{References}

1. Andrés B, Esteban S, Rivera D. Parallel genetic algorithms: an application for model parameter identification in process control. In: Proceedings of EuroPVM/MPI'99, Sabadell (Spain), 1999

2. Aranda J, Cruz JM, Díaz JM, Ruipérez P, Andrés B, Esteban S, Girón JM. Modelling of a high speed craft by a non-linear least squares method with constraints. In: Blanke M, Pourzajani MMA, Vukic, ZZ (eds). Proceedings of the 5th IFAC conference of manoeuvring and control of marine craft 2000. Pergamon Press, 2001, ISBN: 0-08-043659-5.

3. Baitis AE, Appleby TR, Meyers WG. Validation of the standard ship motion program, SMP. Ship motion transfer function prediction, DTRC Report SPD0936-03, 1981

4. Coleman T, Branch MA, Grace A. Matlab optimization toolbox. User's Guide. Version 5. The Mathworks. Inc., 1997

5. Cruz JM, Aranda J, Díaz JM, Ruipérez P. Identification of the vertical plane motion model of a high speed craft by model testing in irregular waves. In: Kijima K, Fossen TI (eds). Control applications in marine systems a proceedings volume from the IFAC conference. Pergamon Press, 2000., pp 257-262, ISBN: 0080430333.

6. Davids L. Handbook of genetic algorithms. Van Nostrand Reinhold, 1991

7. Díaz JM. Identificación, modelado y control de un buque de alta velocidad. Tesis doctoral. Dept. Informática y Automática. Facultad de Ciencias UNED (Spain), 2002

8. Esteban S, Girón-Sierra JM, Cruz JM, Andrés B., Díaz JM, Aranda J. Fast ferry vertical accelerations reduction with active flaps and t-foil. In: Proceedings of 5th IFAC conference on manoeuvring and control of marine crafts MCMC2000. Aalborg, 2000

9. Fossen TI. Marine control systems: guidance, navigation and control of ships, rigs and underwater vehicles. Marine Cybernetics AS, Trondheim, 2002

10. Goldberg DE. Genetic algorithms in search, optimization and machine learning. Addison-Wesley, 1989

11. Hoff O, Kvalsvold J, Zhao R. Global loads on highspeed catamarans. PRADS'92, Newcastle, UK, 1992

12. Holland JH. Adaptation in natural and artificial systems. The University of Michigan Press, Ann Arbor, 1975

13. Korvin-Kroukovsky BV. Theory of seakeeping. SNAME, New York, 1961

14. Lewis, EV. Principles of naval architecture second revision. volume III. motions in waves and controllability. Published by The Society of Naval Architects and Marine Engineers. 601 Pavonia Avenue, Jersey City, NJ, 1989

15. Lloyd ARJM. Seakeeping: ship behaviour in rough weather. Ellis Horwood, 1998

16. Nestergad A. Motions of surface effects ships. Det norkse Veritas (DnV), 1998

17. Michalewicz Z. Genetic Algorithms + Data Structure$\mathrm{s}=$ Evolution Programs. Third, revised and extended edn. Springer-Verlag, 1999

18. Schoukens J, Pintelon R. Identification of linear systems. Pergamon Press, 1991

19. Ursell F. Wave generation by wind. In: Batchelor GK, Davies RM (eds). Surveys in Mechanics, Cambridge University Press, England, 1956, pp 216-249 